\title{
Cities, the Urban Green Environment, and Individual Subjective Well-Being: The Case of Milan, Italy
}

\author{
Giorgio Tavano Blessi, ${ }^{1,2}$ Enzo Grossi, ${ }^{3}$ Giovanni Pieretti, ${ }^{2}$ \\ Guido Ferilli, ${ }^{1}$ and Alessandra Landi ${ }^{2}$ \\ ${ }^{1}$ Faculty of Arts, Markets, and Heritage, IULM University, Via Carlo Bo, No. 1, 20143 Milan, Italy \\ ${ }^{2}$ Department of Sociology and Economic Law, Bologna University, Via San Giacomo 3, 40126 Bologna, Italy \\ ${ }^{3}$ Bracco Foundation, Via Cino del Duca, No. 8, 20122 Milano, Italy
}

Correspondence should be addressed to Giorgio Tavano Blessi; g.tavano@iuav.it

Received 2 March 2015; Revised 2 May 2015; Accepted 29 June 2015

Academic Editor: Leiwen Jiang

Copyright (C) 2015 Giorgio Tavano Blessi et al. This is an open access article distributed under the Creative Commons Attribution License, which permits unrestricted use, distribution, and reproduction in any medium, provided the original work is properly cited.

This paper evaluates the independent effect of the spatial proximity of green urban areas upon the individual subjective well-being of the Milan population (Italy). The methodology is based on a survey undertaken in 2010 using a sample of 1,000 of Milan citizens. Univariate and multivariate analyses and GIS localization have been employed in order to rank the major individual well-being determinants and the relationship between citizens and urban green areas. Results show that the residential proximity of citizens to urban green areas seems to have little bearing on individual subjective well-being.

\section{Introduction}

In the last decade, a growing flow of literature has pointed out the role of urban provision in terms of tangible urban assets, such as the built and green urban environment, and intangible urban assets, such as knowledge, relationships, and culture, in promoting the society development and enhancing social welfare. With respect to social welfare, the debate in the last decade has focused attention on the quality of life of individuals and the urban environment and how the quality and quantity of built and green urban environment can affect human development, highlighting the impact in relation to the areas of health and subjective well-being. Although previous studies concerning the impact of green urban areas on individual well-being have added greatly to our knowledge of the role of the natural urban landscape on individual health, little is known about the effect of the spatial proximity of citizens to the urban natural environment and the impact of this element on subjective well-being. Even if it is quite understandable that living near a green area should provide more benefit in terms of perception of quality of life, and thus subjective well-being, rather than near an industrial site, the majority of the analyses have focused on the impact of green areas, with the role of these spaces in leisure and social activities, accessibility, and usage, and therefore on the effect on the health and subjective individual well-being with the participation in such activities. Little attention has been directed to the effect of living in proximity to green urban areas on psychological well-being in general terms, and the results have been controversial. On the one hand, a study undertaken in London in 2009 found that the proximity of urban green spaces bears no relation to the subjective well-being of city residents [1]. On the other hand, research promoted in China in relation to proximity to urban green area found a significant relationship between this availability and individual well-being [2].

The paper aims to evaluate the relative importance of green urban areas and in particular public parks and gardens located in the heart of the city of Milan, challenging the assumption that proximity to green space is significant in relation to the stock of various features that in the literature are assumed to be possible determinants concerning individual urban subjective well-being. In particular, the focus will be on the relationship between the spatial proximity of 
city dwellers and green areas, suggesting a range of modes in which these could be associated with social well-being and so considering the results of urban planning and the management of the urban green environmental area as a possible tool for proactive welfare policy.

The contents of this paper are organized along three basic lines. In the first part, we present a review of the relevant literature on well-being and then emphasize the relationship between the urban built and green environment and subjective well-being. In the second part, we present and analyze the results of the survey undertaken and finally, in the third part, we show the results and draw some conclusions.

The analysis is based on a survey based on a statistically representative sample of the Milan population and undertaken in the winter of 2010. The survey collected a wide range of information in order to highlight the incidence of individual sociodemographic features as well as investigate to what extent the amount of green areas can be employed as a key explanatory factor in relation to subjective well-being [3]. It is important to deepen our knowledge of these associations in order to provide useful recommendations for planning and the implementation of new services and strategies in relation to individual and social well-being development within urban areas.

\section{Well-Being Considered on the Grounds of the Theory}

Studies on individual and societal well-being conditions arose in academia in the 1970s [4], a time when it is possible to observe a progressive shift of researchers' attention from the evaluation of the impact of elements related to "individual human needs" on individual and societal development, such as food, shelter, and welfare services like education and health, to those fostering individual behavior in the real postindustrial scenario of most advanced countries, seen as "individual identity needs." As Offer underlines [5], in the development model of most advanced societies, it actually emerges that behavior of individuals leads to a strategy focusing on the achievement of personal identity and thus on the elements which may foster an improvement in the quality of life, rather than on those related to answering basic needs, which can easily be met by the market [6]. This relevant modification of individual behavior is clearly emphasized in the development dynamics of postindustrial societies, a process which drives individuals toward the exploration of the possible choices for preference satisfaction with the aim of increasing their quality of life and hence their well-being [7]. The main features characterizing individual well-being are attributable to two areas [8]. The first is associated with objective well-being (OWB), which is generally evaluated through indicators relating to the measurement of observable variables, for example, life expectancy, quality of the environment, access to food, the education system, and the welfare state, which individuals see as important elements for the quality of life. The second concerns subjective well-being (SWB), where measurement is taken by asking individuals about their sociodemographic status and subsequently about
TABLE 1: Sociodemographic characteristics of survey sample.

\begin{tabular}{|c|c|c|c|c|}
\hline \multirow{2}{*}{ Sample data } & \multicolumn{2}{|c|}{ Men } & \multicolumn{2}{|c|}{ Women } \\
\hline & $N$ & $\%$ & $N$ & $\%$ \\
\hline \multicolumn{5}{|l|}{ Age } \\
\hline $15-24$ & 59 & 12,39 & 60 & 11,45 \\
\hline $25-34$ & 35 & 7,35 & 56 & 10,69 \\
\hline $35-44$ & 73 & 15,34 & 61 & 11,64 \\
\hline $45-54$ & 111 & 23,32 & 126 & 24,05 \\
\hline $55-64$ & 67 & 14,08 & 63 & 12,02 \\
\hline $65-74$ & 75 & 15,76 & 95 & 18,13 \\
\hline 75 or more & 56 & 11,76 & 63 & 12,02 \\
\hline \multicolumn{5}{|l|}{ Education } \\
\hline University & 116 & 22,3 & 125 & 18,5 \\
\hline High school & 227 & 45,5 & 255 & 46,7 \\
\hline Secondary school & 112 & 27,1 & 117 & 27,2 \\
\hline Primary school & 19 & 4,4 & 37 & 6,6 \\
\hline \multicolumn{5}{|l|}{ Marital status } \\
\hline Single & 161 & 33,8 & 126 & 24 \\
\hline Married & 243 & 51,1 & 285 & 54,4 \\
\hline Widowed & 19 & 4 & 62 & 11,8 \\
\hline Divorced & 30 & 6,3 & 33 & 6,3 \\
\hline Cohabitee & 22 & 4,6 & 15 & 2,9 \\
\hline Not declared & 1 & 0,2 & 3 & 0,6 \\
\hline \multicolumn{5}{|l|}{ Income } \\
\hline 1000 Euros or less & 33 & 6,9 & 62 & 11,8 \\
\hline 1001-1500 Euros & 58 & 12,2 & 62 & 11,8 \\
\hline 1501-2500 Euros & 82 & 17,2 & 79 & 15,1 \\
\hline 2501 Euros or more & 120 & 25,2 & 98 & 18,7 \\
\hline Not declared & 183 & 38,4 & 223 & 42,6 \\
\hline PGWBI (mean I.C. 95\%) & 83 & 1,36 & 78,32 & 1,43 \\
\hline
\end{tabular}

their perception of various elements related to the external environment.

Starting in the late 1990s, studies have focused attention on the determinant of subjective well-being, investigating the reasons why individuals experience their life positively [9]. Focusing our attention on the theory of individual subjective well-being, it is possible to define the latter as a subjective element resulting from the impact of various factors, such as economic, social, and environmental ones, on the individual perception of their lives [10]. As Lahtinen points out [11], these factors can be subdivided into two main dimensions:

(a) The sociodemographic characteristics embedded in an individual-for example, state of health, education, age, gender, and employment (see Table 1).

(b) The environmental factors embedded in the space and place where an individual lives and works, for example, the built environment, climate, transport/commuting, and the natural environment such as public parks and gardens.

Several studies have highlighted the role of sociodemographic factors in relation to perception of well-being (for a detailed review in different academic areas, see for psychology Pavot and Diener [12] and for economics Frey and Stutzer [13]). Concerning the environmental factors 
affecting subjective well-being, studies carried out have tried to evaluate certain elements such as commuting [14], spatial differentiation in terms of access to tangible and intangible urban provision [15], climate [16], cultural provision and infrastructure [17], and the social dynamics relating to the quality and quantity of private and public urban places, such as buildings and open-air spaces present in [18]. A systematic and extensive study on resources and quality of urban life (over 700 indicators for every zip code in the US) has been carried out thanks to Clark et al. [19]. The research project aimed at obtaining a better understanding of how certain amenities, such as music, art, theatre, and restaurants and also parks and beaches, contribute to a community's vitality. Many neighborhood resources are taken into consideration and the authors stress the combination of urban infrastructure such as building and parks along with race, class, gender, and education, in supplying enhanced opportunity in terms of quality of life.

A recent study, which encompasses in a uniquely wider evaluative framework the sociodemographical and environmental factors in relation to happiness in urban areas, has been furnished by Florida et al. [20]. In his analysis, thanks to a most extensive data set supplied by Gallup Poll, Florida et al. have focused attention on the urban environment, providing some interesting pointers with regard to the possible impact of green urban areas upon individual well-being but failing in terms of accuracy, given the fuzzy theoretical approach to happiness and well-being determinants within urban areas.

Despite the extensive literature produced in the last 20 years, the number of researchers investigating the impact of urban green areas on individual well-being is still growing. This also depends on the fact that evidential results are ambiguous. We have built on previous studies [21] which have analyzed the role of different sociodemographic characteristics in the subjective well-being of the Milan population and have carried out an analysis of the spatial proximity relationship between green areas and individuals' perception of well-being, focusing on and challenging the hypothesis of how significant this element is in relation to the classic subjective determinants such as income, education, gender, civil status, employment, and leisure activities within a potential urban well-being scheme.

\section{The Taxonomy between the Urban Built and Natural Environment and Subjective Well-Being}

The relationship between urban environment and individual well-being is a multifaceted matter, a complexity which is driven by the scientific approaches and hence by the evaluation frameworks employed. Several studies have highlighted the different effects of the built environment in relation to individual well-being. Starting from the work of Ulrich et al. [22], which has highlighted the restorative benefits of natural and urban environment on stress recovery, Kuo et al. [23] in their seminal work have emphasized the direct and indirect impact of the built environment on subjective wellbeing. In their study they found a direct correlation between the quality of housing with structural housing type-design, maintenance, and floor allocation-and psychological distress. Apparicio et al. [24] question the impact of new housing insertion in the surrounding urban environment, with particular attention to public housing in the Montreal metropolitan area. Using GIS information and correlating it with individual sociodemographic data and analysis of public and private infrastructure and building, they found that these planning processes are more likely to produce social disadvantage on both preexisting and new residents, given the high level of social deprivation present, the low quality of new building work, and the amount of community and social facilities, which all act on the well-being of individuals.

Other studies have tried to evaluate the impact of the built environment on the social sphere, an important element given the role played by the social atmosphere of a location on the individual perception of well-being. Evans [25] found a correlation between neighborhood characteristics and the mental health of residents, pointing out that it is difficult to separate the quality of housing from the neighborhood context because, for example, poor-quality houses are more likely to be located in peripheral urban areas, which generally see the presence of a social context with several indicators of urban decay. Clark et al. [26] identify other elements of the built environment, which can affect the social dimension and thus individual subjective well-being. These are the transport infrastructure and housing dispersion, which impact on social relationships and social support, given the physical distance between individuals. Punter [27] describes the strategic influence of specific kinds of buildings such as those dedicated to social and cultural activities as possible "social black boxes" [28], which can provide the opportunity for individuals to build social relationships and actively participate in urban life. In this respect, they can also prevent criminality at a microlevel and, at a macrolevel, urban and social degradation. In the same way, specific kinds of infrastructure such as those related to community use can act towards building a sense of community and improving the social sphere, thus promoting individual well-being [29].

If the correlation between the urban built environment and subjective well-being is evident, the association between the urban green environment and well-being is also relevant. On the subject of the effect of parks and gardens on subjective well-being, analyses have focused attention on the positive effects of taking advantage of these places in the social dimension and their influence on the perception of wellbeing. The foundational work of R. Kaplan and S. Kaplan [30] underlines the psychological role that natural environment plays in individual lives, with a specific focus on urban green areas, such as parks, gardens, and its pervasiveness in the people quality of life perception, providing also possible hints for the planning and management of such urban resources. In the same research vein, certain characteristics of green urban spaces such as public access and community use contribute to the engagement of individuals with the natural environment and each other, fostering a sense of place; this plays an important role in prompting social cohesion and identity [31]. Parks and gardens can thus be considered as potential "relational spaces," enhancing relationships and 
connections with other people, all elements that are parts of the wider framework of the social capital of a location in terms of improving individual subjective well-being [32]. In this context Chu et al. [33] show how green areas can be defined as social infrastructures having positive effects on individual well-being, particularly in areas presenting a high density of population and infrastructure. The review of international policy interventions on well-being identified a number of case studies where interventions promoting green space (parks, community gardens, and allotments) had led to well-being benefits [34]. These included improved communication between social groups, increased feelings of self-worth, greater sense of community, relaxation, and increased physical health.

In relation to the perceived availability, accessibility, and visual proximity to green areas by urban-dwelling citizens, studies have shown the importance of such elements as spatial proximity in relation to subjective well-being.

Gildlöf-Gunnarsson and Öhrström [35] analyzed how the perceived availability of nearby green areas affects individual well-being. Thanks to a cross-sectional survey undertaken in residential areas of Stockholm and Goteborg, it was found that the close availability of and easy access to green urban areas reduce the psychological and social distress symptoms of individual well-being and increase in individuals the willingness to take advantage of outdoor spaces.

In terms of accessibility to urban green areas, Pretty et al. [36] work on the impact of access to green space on subjective well-being. In the study the authors identify the importance of the conservation of such areas as parks and gardens located in urban areas. Pretty et al. recognize a framework of different features such as physical constraints (roads and distance) and also cultural and social factors which can act as potential restrictions on a willingness to go to urban green areas. Giles-Corti et al. [37] investigate the correlation between access to POS (public open space) such as parks and gardens. Following a survey undertaken in Melbourne, the authors found that green areas with very good access, location (proximity to citizens), and size are $50 \%$ more likely to attract individuals and to create advantages in the areas of health and subjective well-being.

In conclusion, the relation between visual proximity to urban green areas and individual subjective well-being has been investigated from Kaplan [38]. Thanks to a research undertaken on a sample of six low-rise apartment communities, the view of natural elements, such as urban green areas, from the dwellers apartment will greatly affect the individual satisfaction in relation to the perceived quality of the neighborhood, promoting positive impact on individual well-being perception.

\section{Some Recent Evidence from Empirical Studies: The Case of Milan}

During the winter of 2010, a cross-sectional survey of a statistically representative sample of the Milan population was carried out (1,000 people), stratified according to neighborhood, place of residence, and population size in relation to individuals aged 15 years or above. This survey set out to understand the impact of a number of determinants of a sociodemographic, cultural, and environmental matrix on the well-being of residents in the municipal area. The survey was conducted with the assistance of Doxa, an Italian pollster company, through telephone interviews, according to the CATI system.

The methodology consisted of drawing up a questionnaire based on three specific areas:

(a) A questionnaire specifically aimed at the evaluating and assessing of subjective well-being employing PGWBI, an index that has been validated by decades of clinical practice and in its full version (22 items). The PGWBI consists of 22 self-administered items, rated on a six-point scale, which assess the psychological and general well-being of respondents in six domains: anxiety, depressed mood, positive wellbeing, self-control, general health, and vitality. Each item has six possible scores (from 0 to 5), referring to the last four weeks of the subject's life. Each domain is defined by a minimum of 3 to a maximum of 5 items. The scores for all domains can be summarized in a global summary score, which reaches a theoretical maximum of 110 points, representing the best achievable level of well-being, a sort of "state of bliss." The instrument is a widely used scale across many conditions and as underlined by Lundgren-Nilson et al. [39] it has quality, satisfactory internal construct validity when tested with modern psychometric techniques, that would supply reliable outcomes in the well-being dimension.

(b) Sociodemographic and health-related data which are widely recognized as relevant determinants of wellbeing are residence area (address and zip code in order to use the GIS system and locate their position in relation to the city green area), gender, age, schooling, civil status, monthly income level, and the presence/absence of a given list of diseases.

(c) 15 different variables relating to cultural access have been added following a scrutiny of the relevant literature in the cultural field; such variables are to be jointly meant as a proxy of individual levels of "cultural access."

The Case Study: The Milan Urban Area. Milan is the second largest city of Italy, located in the northern area of the country. The number of residents based on data of 2011 provided by the Milan Bureau of Statistic was 1,262,101 in an area of $182,07 \mathrm{~km}^{2}$. In the ranking of the most populated Italian urban areas (over 60,000 habitants), it is possible to observe that Milan ranks in second place at national level in terms of resident density behind Naples, with 6,932 citizens per square kilometer (Italy 198,78; source ISTAT (Italian Statistical Bureau), 2013 from 2011 data). With respect to the Milan green area, including public parks and gardens, the data provided for the Milan Municipality show that an average of $11.6 \%$ of the territory is allocated as green spaces (the median value for Italian urban areas with more 
TABLE 2: Park list.

\begin{tabular}{lc}
\hline Park name & N map \\
\hline Parco Sempione & 1 \\
Parco Solari & 2 \\
Parco delle Basiliche & 3 \\
Parco Marinai d'Italia & 4 \\
Giardini Indro Montanelli & 5 \\
Parco Guido Galli & 6 \\
Parco Nord & 7 \\
Parco Lambro & 8 \\
Parco Forlanini & 9 \\
Parco di Trenno & 10 \\
Boscoincittà & 11 \\
Parco Monte Stella & 12 \\
\hline
\end{tabular}

than 60,000 residents, $14.9 \%$; source ISTAT (Italian Statistical Bureau), 2013 from 2011 data), and green space availability per resident is about $16,3 \mathrm{~m}^{2}$ (the median value for Italian urban areas with more than 60,000 residents, $30.9 \mathrm{~m}^{2}$; source ISTAT (Italian Statistical Bureau), 2013 from 2011 data). On the basis of the data presented, it is possible to observe the scarcity of available urban parks and gardens in the municipal area of Milan, which exacerbates the possible effect depicted by Anderson and West [18] of a rise in the perceived social and well-being value of parks in very dense, populated cities.

Milan metropolitan area documents report 78 gardens and parks. The 12 green areas considered in our study are only parks, and not gardens, and have been selected in terms of dimensions and attractiveness and on the base of the following criteria:

(i) distribution among those within the historical Milan area (from number 1 to 6 ) and those located in the metropolitan ring (from number 7 to 12 );

(ii) size of parks and the amenities located therein;

(iii) number of annual attendances taken from information provided by the Milan City Council;

(iv) staging of events within the parks (cultural, sporting, and social).

The list of parks with a map indicating the exact location is provided in Table 2 and Figure 1.

\section{The Results}

In this section, the sampling results will be presented and some remarks will be outlined about the main determinants of individual subjective well-being as identified by the literature in the subject. As a result, a ranking of variables having the greatest impact on the respondents' well-being will be made on a gender basis. As a final element, certain evidence on the relationship between green areas and place of residence will be presented in order to assess the impact of all the determinants considered for the construction of personal well-being.

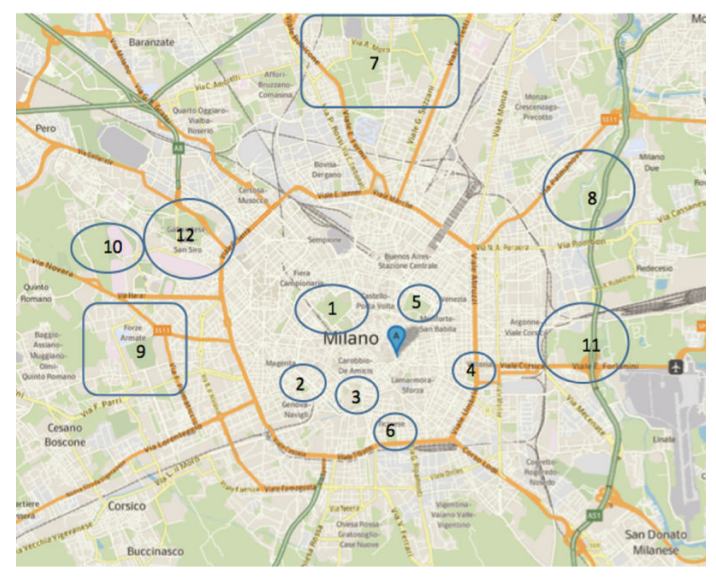

FIGURE 1: Milan urban area and parks.

Starting from an overview, what we can see from this analysis is substantial psychological well-being in the Milan population. The data, indeed, report that the average wellbeing level is positive in men and women, as has also emerged in previous studies [21]. By going into greater depth, that is, through analyzing the incidence of the main determinants of individual well-being, we also can see how this analysis substantially reflects the literature of the subject (Figures $2(\mathrm{a})-2(\mathrm{~g}))$.

At a general level, our analysis of the main determinants by gender confirms what has emerged in other surveys [17] as well as what emerges from the literature about well-being described in Section 3: women's perceptions of happiness are lower, even in bivariate correlation with determinants like age or education. Looking in detail, we can confirm a substantial similarity between our sample and what the literature reports [21]. Regarding age, the curve indicates that a decline in well-being from 20 to 30 tends to return at a higher level and stabilize over time. Income in particular affects men's perception of well-being, signaling once again that this element becomes a "marker of identity" of personal value in relation to professional position. With regard to employment, we can see that jobs of high responsibility and autonomy (e.g., entrepreneurs and managers) produce a stronger sense of well-being if compared to employees, teachers, or craftsmen. The data concerning the incidence of marital status also confirm the literature on the subject; marriage produces higher well-being values for men but, in the case of separation, these dramatically decrease (by almost 10 points). Educational qualifications are directly correlated with well-being, with a relevant positive curve with regard to women. With regard to family composition, it affects men and women equally; as we can see, indeed, having a higher number of offspring allows well-being to increase. We also considered the absence or presence of disease as a determinant and we can see its strong influence on the perception of well-being. In this case, the curve shows a negative trend as the amount of diseases increases; this is especially true when these are concomitant, degenerative, or chronic. 


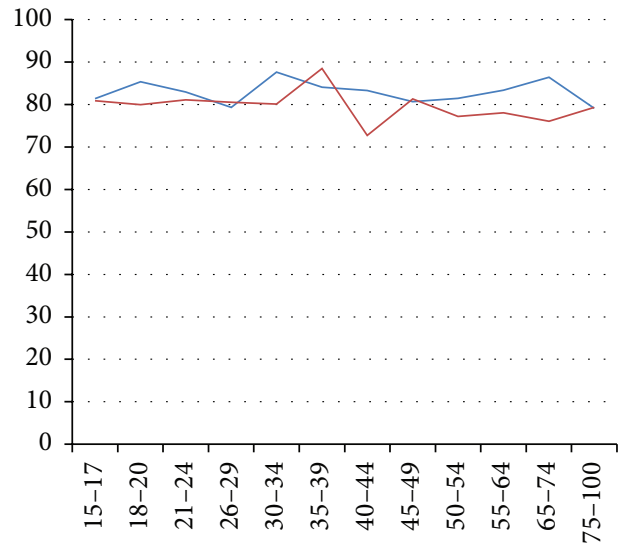

Men
_ Women

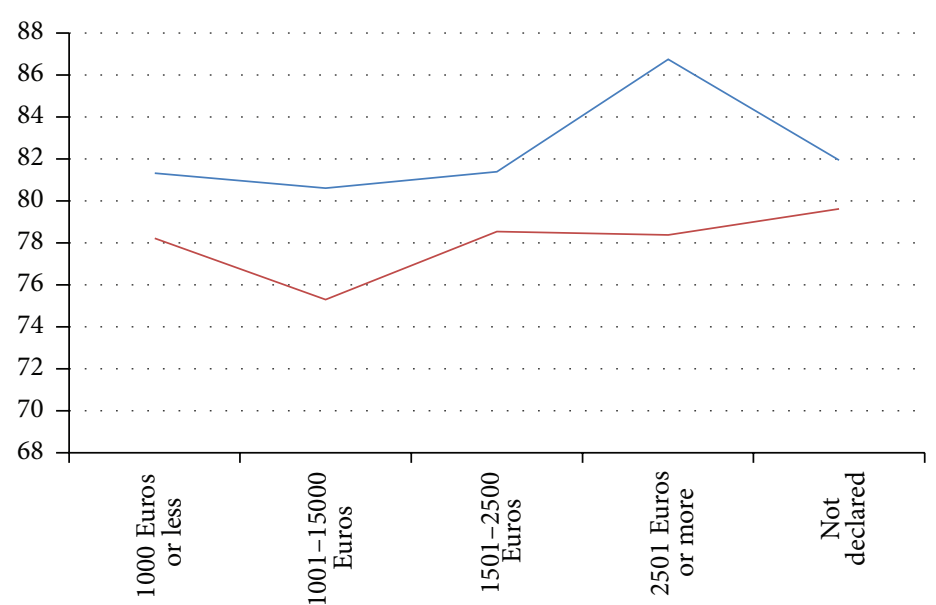

(b) Income
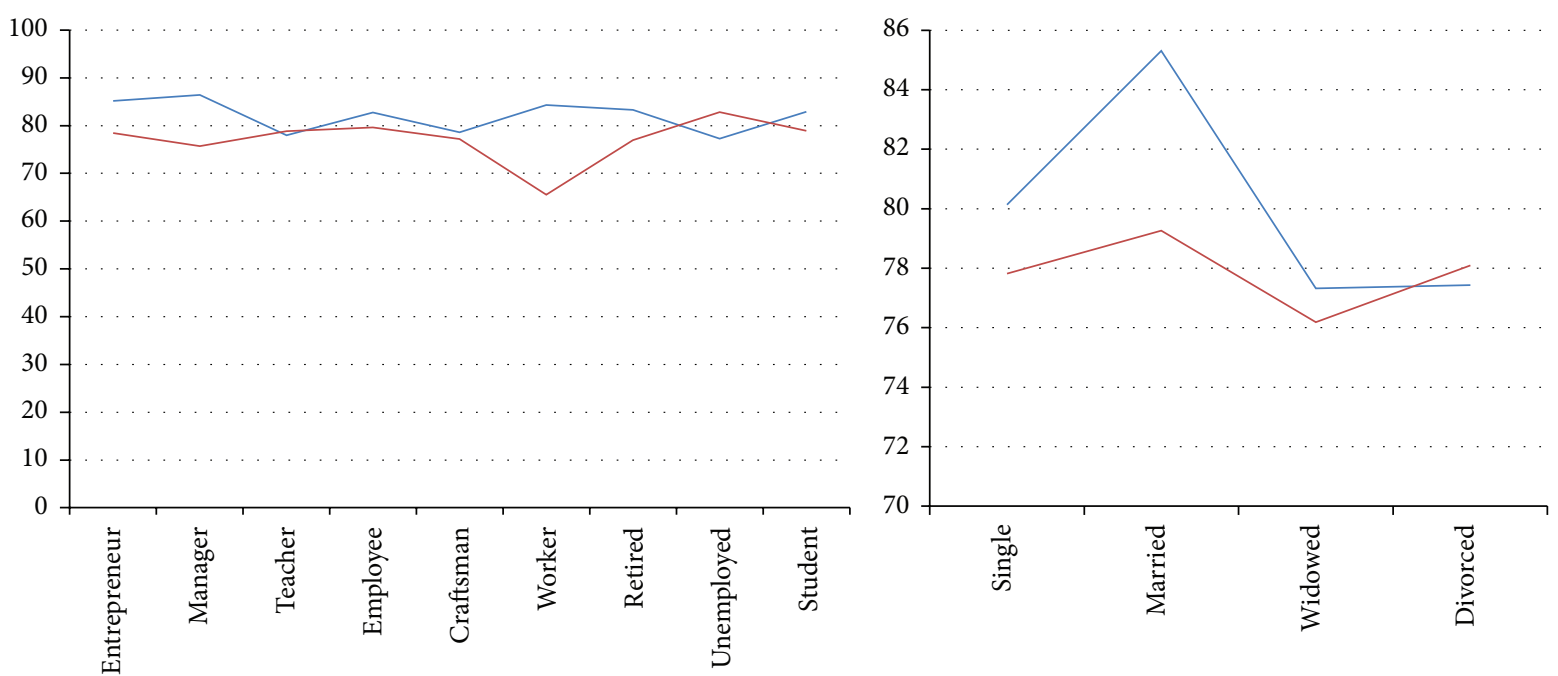

- Men

— Women

(c) Occupation

(d) Marital status
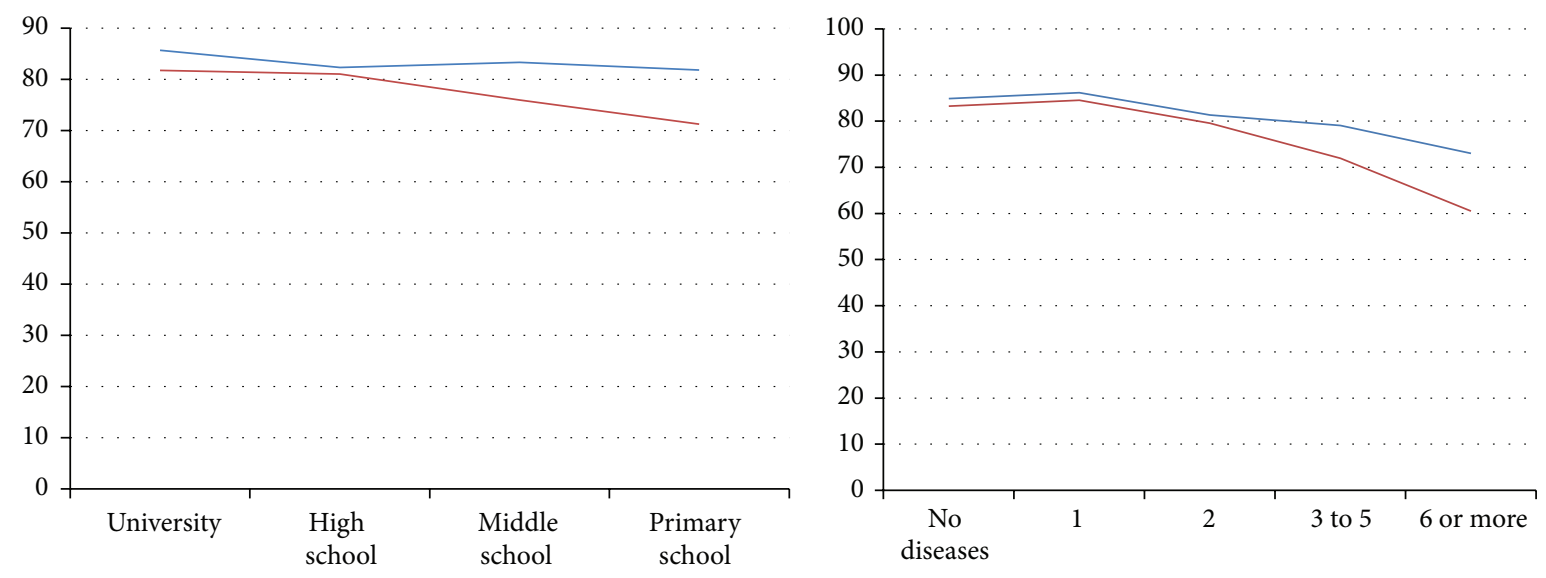

- Men

- Women

(e) Education 


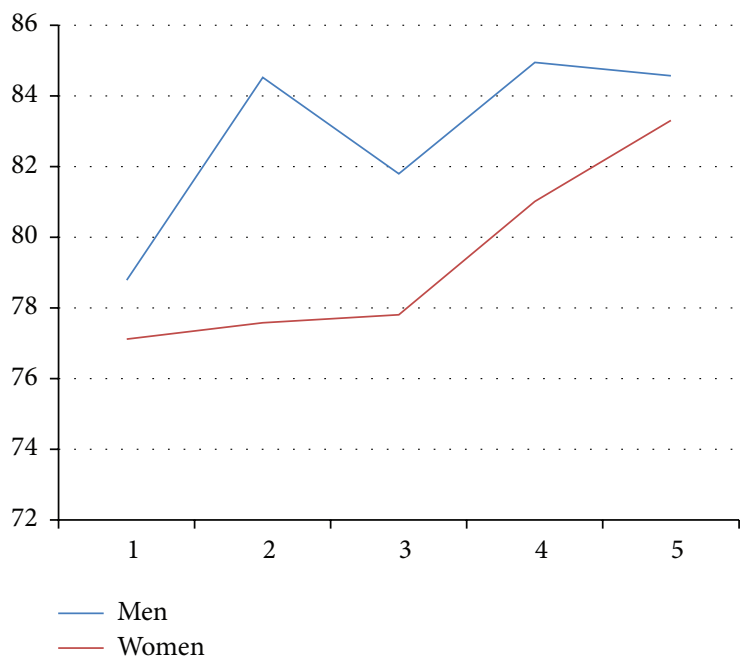

(g) Family members

FIGURE 2: Level of perceived well-being (PGWBI) in relation to the main sociodemographic sample variables.

A second level of analysis is related to the ranking of variables that compose the basket of perceived positive wellbeing factors. In this case too the distribution was carried out on a gender basis (see Table 3 ).

The ranking provides an interesting insight into the value assigned to determinants by men and women. In general, we can observe the evident importance of spare time (e.g., all the cultural or recreational elements such as physical activity) in relation to the positive perception of well-being, together with education. With regard to gender comparison, we also point out the difference connected to marital status. Marriage seems to have a crucial impact on male well-being; once again, the results of previous studies on this topic are confirmed [40].

As a final step, the spatial proximity to green areas with regard to individual subjective well-being was considered. Even if their contribution is perceived as very significant by public opinion, as we can see from the literature review referred to in the introductory section of the paper, the role of individual spatial proximity to green areas in subjective wellbeing is ambiguous.

The first investigative level consisted of evaluating the relationship between well-being ( $y$-axis) and the distance from green areas to the place of residence of our sample respondents ( $x$-axis) of Figure 2. For each individual part of the survey, the distance was measured from the 12 parks, employing as measurement criteria the straight-line distance between home and the green area. Well-being was measured through the rating scale we adopted as an assessment tool: the PGWBI ( $x$-axis in Figure 2). As we can see from Figure 2, the dispersion trend line of points is essentially flat, which means that no relationship between subjective well-being and proximity to green spaces can be inferred, and distance has an irrelevant impact on individual well-being (see Figure 3).

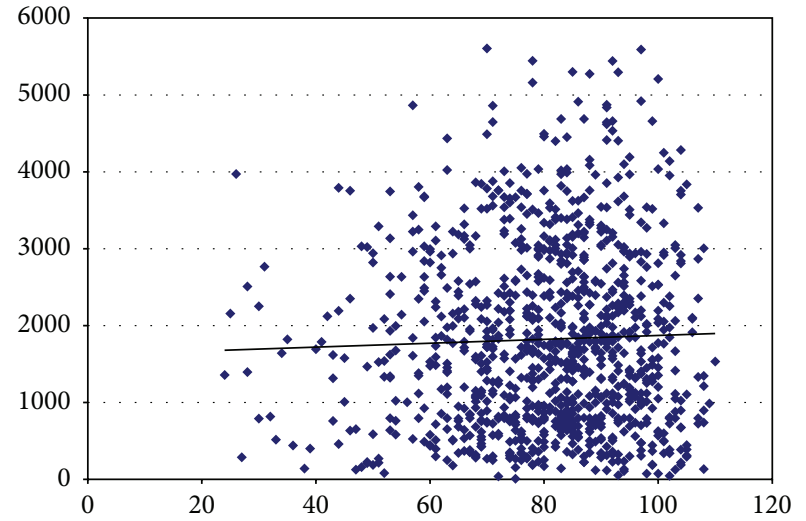

FIGURE 3: Dispersion of points and trend line of individual wellbeing in relation to the distance between green areas and place of residence. Correlation index of the regression is equal to 0.033 .

The same footprint of analysis was carried out at a further level; we grouped all the information from the sample into classes. In this way, the clustering process led to nine classes with regard to distance ( $x$-axis) from less than 200 meters to more than 3 kilometers between green areas and place of residence and PGWB level ( $y$-axis). We did so on the base of previous studies that reported these data as a constraint, a threshold below which green areas generate a higher potential contribution to individual well-being [41].

Figure 4 illustrates how classes move, showing two interesting sets of data with respect to the research topic of this paper. Let us make a first general consideration; there is a small or no difference in the perception of well-being. For example, the first class, consisting of people who live less than 200 meters from a green area, is essentially equal 
TABLE 3: Ranking of principal positive well-being determinants on a gender basis.

\begin{tabular}{lccc}
\hline \multicolumn{1}{c}{ Men } & \multicolumn{2}{c}{ Women } \\
Variable & $r$ & Variable & $r$ \\
\hline Married & 0,21 & High cultural frequentation & 0,25 \\
High income & 0,16 & Income & 0,19 \\
Visiting a museum & 0,14 & Education & 0,18 \\
Physical activity & 0,14 & Going to the cinema & 0,17 \\
High cultural frequentation & 0,12 & Visiting a museum & 0,15 \\
Regular physical activity & 0,11 & Reading novels & 0,14 \\
Education & 0,09 & Regular physical activity & 0,12 \\
Reading novels & 0,08 & Theatre attendance & 0,11 \\
\hline
\end{tabular}

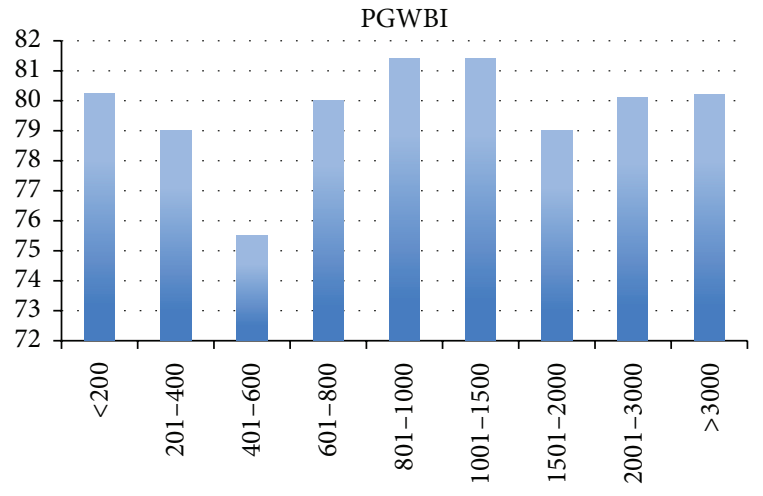

FIGURE 4: Impact on the perceived well-being in relation to the distance from the closest green area.

to that of people who live more than 3000 meters away. A further consideration is limited to the first five classes which represent the people who live no more than 1000 meters from green areas. Our results show that the perceived well-being tends to proportionally decrease up to 600 meters distance and then gradually returns to the initial level and tends to stabilize, as we pointed out before.

A third level of analysis has been undertaken considering the previous one, focusing not on the whole sample but on those effectively living beside parks and gardens. Using GIS localization, we have correlated the information concerning walking distance to the green space and perceived well-being. The threshold adopted was 500 meters walking distance between urban dwellers and each natural area. The results are shown in the chart and table of Figure 5 and Table 4 . As it is possible to observe, the PGWBI levels of those living in the surrounding area of parks and gardens differ substantially. The urban dwellers living near a green area located in the historical center of Milan (ID number from 1 to 6) show a PGWBI level above the median value of the whole sample $(80,2)$, with a peak in the case of the Parco delle Basiliche. In contrast, Milan residents living close to parks and gardens outside the historical center (ID number from 7 till 12) display a level that is considerably below the rest of the population. In order to demonstrate the influence of green urban areas on the full spectrum of subjective well-being determinants,

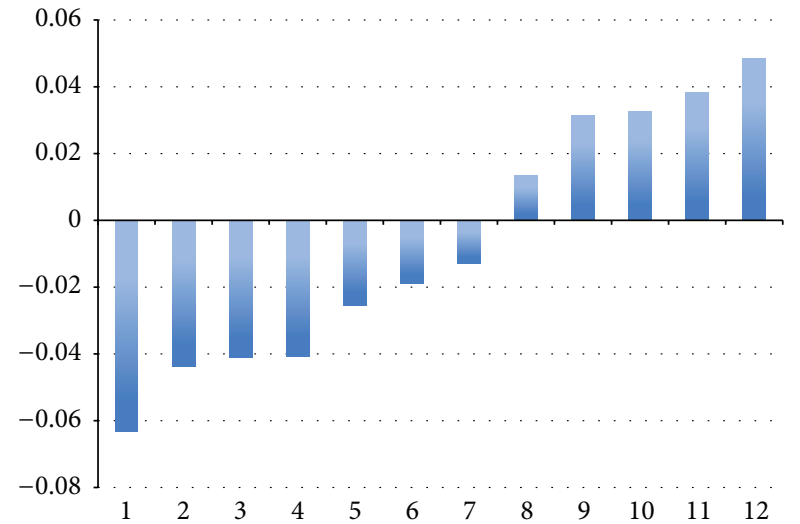

FIGURE 5: Linear correlation between distance to the green area and PGWBI.

we have provided a linear correlation between distance to urban green areas and PGWBI. In this case, a positive value indicates that the greater the distance between the individual and the park is, the lower the effect of living near it will be. On the other hand, a negative value illustrates that the closer the individual lives to the park, the greater the perception of well-being in relation to that space will be. Furthermore, we adopt the coefficient index of the variables (distance to green areas), in relation to the PGWBI.

Data paint a picture in which the green areas located within the historical center seem to add more benefit to the quality of life compared to those outside it, but the relevance in terms of impact is fairly low (see Figure 5 and Table 4).

A final level of analysis was conducted to check the scale of incidence of all determinants detected through the sampling, including the size of green areas (Figure 6), through multivariate analysis.

Multivariate analysis has been carried out with supervised artificial neural networks, according to the method already adopted in Buscema [42]. The choice of a relatively unusual and sophisticated inferential technique such as artificial neural networks (ANNs) is motivated by the fact that the underlying relation to be estimated among our independent sample variables and the dependent variable (the PGWB total score) is extremely complex and there is no reliable a priori statistical model to refer to. ANNs self-adjust their 
TABLE 4: Coefficient index between distance to the green areas and PGWBI.

\begin{tabular}{|c|c|c|c|c|c|c|c|c|c|c|c|c|c|}
\hline & 7 & 8 & 1 & 9 & 2 & 10 & 3 & 4 & 11 & 12 & 5 & 6 & PGWBI \\
\hline 7 Parco Nord & 1 & & & & & & & & & & & & \\
\hline 8 Parco Lambro & 0.49 & 1 & & & & & & & & & & & \\
\hline 1 Parco Sempione & 0.31 & 0.20 & 1 & & & & & & & & & & \\
\hline 9 Parco Forlanini & 0.06 & 0.86 & 0.21 & 1 & & & & & & & & & \\
\hline 2 Parco Solari & -0.31 & -0.28 & 0.76 & -0.02 & 1 & & & & & & & & \\
\hline 10 Parco di Trenno & 0.04 & -0.74 & 0.22 & -0.86 & 0.34 & 1 & & & & & & & \\
\hline 3 Parco delle Basiliche & -0.26 & 0.09 & 0.77 & 0.39 & 0.89 & -0.07 & 1 & & & & & & \\
\hline 4 Parco Largo Marinai d'Italia & -0.06 & 0.68 & 0.47 & 0.91 & 0.34 & -0.67 & 0.70 & 1 & & & & & \\
\hline 11 Boscoincittà & -0.03 & -0.80 & 0.12 & -0.89 & 0.28 & 0.99 & -0.14 & -0.72 & 1 & & & & \\
\hline 12 Monte Stella & 0.32 & -0.52 & 0.40 & -0.72 & 0.32 & 0.94 & -0.04 & -0.55 & 0.89 & 1 & & & \\
\hline 5 Giardini Indro Montanelli & 0.32 & 0.74 & 0.74 & 0.78 & 0.37 & -0.43 & 0.67 & 0.88 & -0.52 & -0.21 & 1 & & \\
\hline 6 Parco Guido Galli & -0.12 & 0.72 & 0.28 & 0.97 & 0.16 & -0.80 & 0.55 & 0.97 & -0.83 & -0.70 & 0.78 & 1 & \\
\hline PGWBI & 0.05 & 0.01 & -0.01 & -0.02 & -0.04 & 0.03 & -0.06 & -0.04 & 0.03 & 0.04 & -0.02 & -0.04 & 1 \\
\hline
\end{tabular}

structure as they learn from their own errors and can handle simultaneously a very high number of variables, irrespectively of their underlying degree of nonlinearity, and this leads to structurally robust results even when the underlying statistical process is not well understood, thereby allowing coping with many sources of inferential inaccuracy such as outliers, collinear interactions among variables, and hidden variables [43].

In particular, we work with the family of supervised ANNs, that is to say, with ANN, that address problems where an external, objective target output can be fixed, so that they learn by examples (the training set, i.e., a suitable subsample of the whole database), calculating an error function during the training phase and adjusting the connection strengths in order to minimize the error function until a satisfactory and stable level of accuracy in the prediction/classification task is reached. This type of ANNs thus computes a function of the form: $y=f\left(x, w^{*}\right)$, where $x$ is the input, $y$ is the output, and $w^{*}$ is the set of ANN weights (the function parameters) that encode the ANN's approximate reconstruction of the structure of the function.

In other words, this analysis provides us with what we may call the "input relevance" of each variable. Input relevance is expressed as a quantity of an arbitrary scale proportional to the importance of the contribution of that variable to the implicit statistical model. The higher the value, the higher the importance of the variable (see Figure 6).

The analysis we conducted clearly shows that the incidence of spatial proximity of individuals to green areas (distance to parks in Figure 6) within the basket of well-being determinants is almost nil; while state of health plays a very important role in the perception of individual well-being of our sample, culture is in second place (culture is understood here as participation and enjoyment of cultural events and services). Income seems to have little impact, if not for those having a monthly salary net of more than 2.000 Euros, even if it appears important in other surveys conducted with the same method [21]. In the case of Milan, however, income exceeds the national average threshold, and it does not seem as critical as in other urban areas [44]. Distance between green areas is in the bottom of this ranking.

\section{Discussion and Final Remarks}

The paper has been structured around the contribution of natural urban assets such as parks and gardens to individual subjective well-being, focusing on the relationship between spatial proximity of urban dwellers and green areas, providing a possible ranking of the importance of such elements in comparison with the classic characteristics influencing individual subjective well-being. There are many reasons for studying and promoting city dwellers' well-being, particularly given the percentage of the European population living in urban areas, which reaches $70 \%$ [45]. It is thus critical to understand the impact of urban infrastructural elements such as parks and gardens, and the reasons fall within the great intrinsic value of the natural environment for human living conditions, a value which is generated by and reflects the equilibrium between the individual and the environment in a broad sense.

In this respect, the importance of green urban areas on individual subjective well-being is deeply rooted in collective social function and individual needs. Material needs call for the gathering of private goods and services supplied by the market to satisfy personal wants and needs, whereas social needs are generally fulfilled by a mix of private and public goods and services, most of which are socially provided and enjoyed, with the aim of satisfying social needs and wants such as a connection to others, recognition, friendship, and sense of belonging [46]. The answering of material needs does not generally require noninstrumental interaction, whereas the meaning itself of social needs is most often destroyed in the absence of noninstrumental interaction, so that the market typically fails in securing the latter [47]. Social needs cannot be satisfied in the market since the very fact of buying or selling connections, recognition, or friendship totally alters the nature and implications of such relationships. Thus, the more important this dimension of green urban areas 


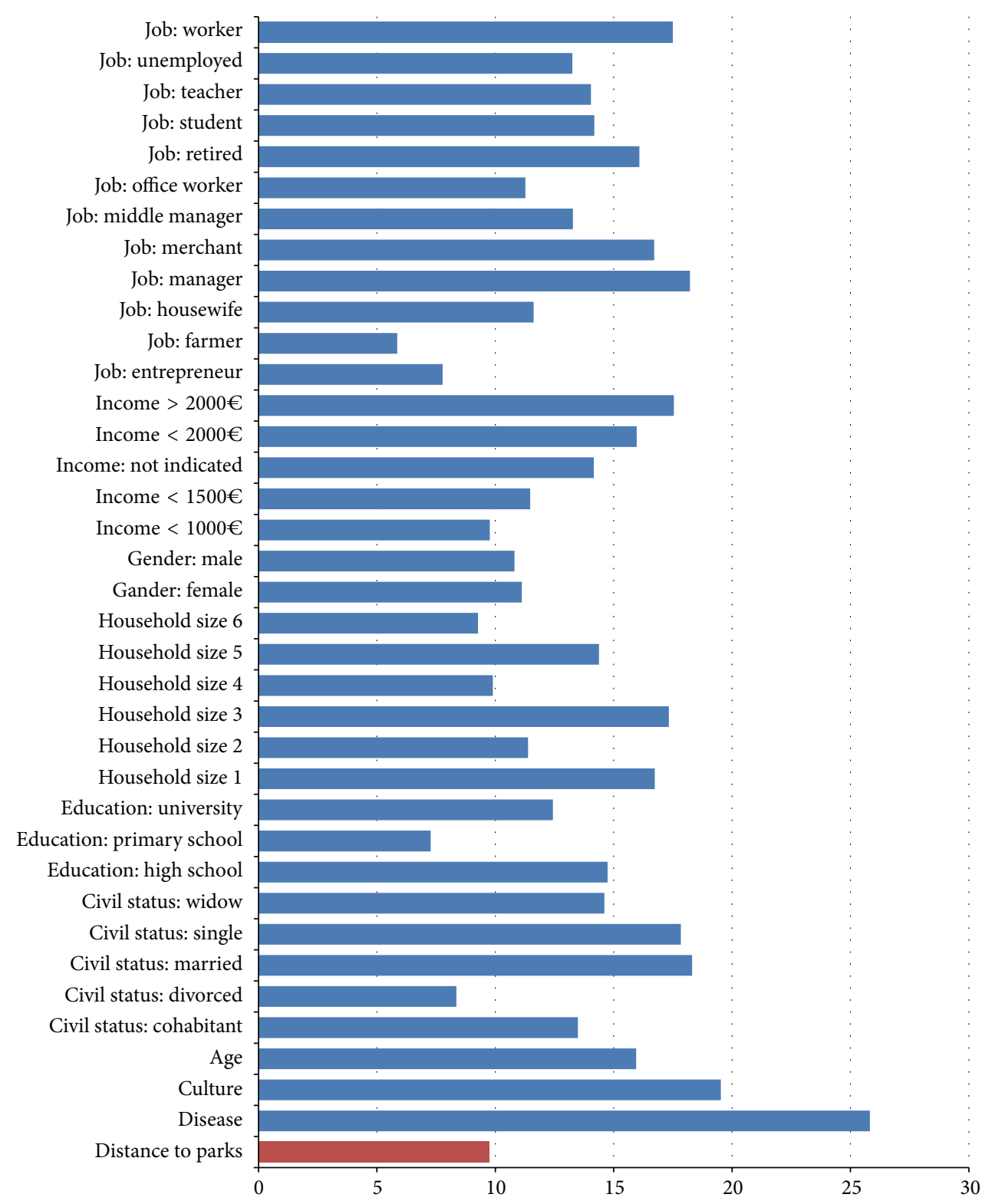

FIGURE 6: Input relevance of urban green areas and sociodemographic variables in multivariate analysis of PGWBI.

becomes for individual and collective well-being, the more socioeconomically advanced societies will have to resort to mechanisms and platforms other than the market to address it properly, in order to generate an efficient level of social welfare and thus well-being [48]. The relationship between urban green areas and city dwellers has been investigated by various disciplines: from the increasing body of economic literature that has analyzed the value of urban green space (either as contingent valuation or hedonic valuation), which has not been mentioned in the theoretical section given the aim of the paper which was direct to evaluate the impact of urban natural environment on psychological well-being, with regard to medicine in relation to physical and psychological health, for example, or sociology in relation to the incidence of structuring relationships and ties of trust to the promotion of well-being and the construction of a larger stock of social capital.

As previously shown, these and other studies reported in the present study have produced evidence of the impact of urban parks or gardens insofar as the usage and/or the accessibility to these urban areas have a positive impact on the individual quality of life. One can certainly take advantage of an urban park with picnic areas for physical activity and to improve one's state of health. Moreover, enjoying an open and exclusive social space can become a factor in promoting relationships and the structuring of social capital as well as individual well-being. Sampson talks about collective efficacy [49] and this concept is used, for example, to understand why parks are resources in some neighborhoods and are seen as dangerous areas in others. At the same time, Small 
dedicates great attention to O' Day Park in his seminal study [50]. Places like this are often seen as dangerous and are greatly stigmatized, but they are also fundamental for local communities as they represent part of their identity, history, and culture.

Let us now summarize the main conclusions of our research undertaken in Milan. Our study was pursued in order to ascertain how spatial proximity to green areas would affect subjective well-being, to what extent these spaces may be able to provide and support the individual, in the hypothesis that spatial proximity will be a possible important element in relation to subjective well-being, particularly in a very high density and polluted urban area as are many European cities such as Milan, in which it is assumed that the need for green areas will be higher rather than for those living in a rural environment. In an urban spatial planning perspective, it is important to provide a comprehensive framework for the role of green urban amenities, not only in the "useable" perspective, but also in terms of individual perceptions and thus of their contribution to psychological well-being.

As noted from the results, which are similar to the London research quoted in Section 2, accessibility to green spaces does not seem to matter much for Milanese citizens. This could be explained in a very simple urban economics framework, where people with different preferences on green versus historical and cultural amenities choose to live in different parts of a city (historical inner cities versus more recent and green suburbs). Furthermore, here we record a counter-intuitive effect. If the relationship between visiting a green urban space and a condition of distress would seem relevant, in our case the relationship between the individual spatial proximity to parks and urban natural environment is a weak one. The counter-intuitive finding that living near green spaces is noninfluential on average in psychological well-being can be explained in several ways, for example, the fact that an undefined subset of people might have decided to live nearer to a park for the very reason that they were distressed. People affected by mild depression or anxiety feel more comfortable in these kinds of city areas surrounded by parks. As with many other salient economic decisions, such as the construction of an investment portfolio, the purchase of a home is made over days or weeks, not in seconds, and is influenced by one's affective state. Therefore, the presence of a subset of people, who, for example, are depressed and live near a park, can easily affect the mean value of the PGWB index, counterbalancing higher values of people who actually benefit from the presence of a green environment.

There is evidence from some studies that being close to a park, on the other side of the road from it, is an amenity for some but is a disamenity for others. In other words, the whole question of the role green spaces and parks play in everyday life and feelings of well-being is complex and highly variable between city dwellers. Individual well-being with respect to distance to urban green areas, such as parks and gardens, is subject to positive feedback dynamics of selfreinforcement based on the acquisition of specific health and psychological elements, which can be determined by direct and personalized preference and perceptions. In this perspective, individual characteristics will affect the perception of individual quality of life in relation to the presence or absence of green areas, an element which has been foreseen, for example, in the research of Nielsen and Hansen. Nielsen and Hansen [51] have tried to evaluate the impact of green areas on stress and obesity. The study reports that the number of visits, meaning access to green areas, cannot explain the effects of these elements on the health indicators. It is suggested that the significance of distance to green areas is mainly derived from its correlation with the intrinsic, sociodemographic characteristics of the neighborhood and its conduciveness to possible outdoor activities and healthy modes of life.

To identify whether and how accessibility and usage are of importance is likely to involve a complex set of issues needing exploration and would require talking to people in a less structured way than a questionnaire allows.

The challenges from using this particular combination of methods in this study are related to the sample size, which is statistically representative, the methodological criteria used for sample stratification, the robustness of the wellbeing index employed, the possibility to correlate several dimensions of individual behavior such as cultural participation and health status, and the power of the inference engine, based on artificial neural networks.

The lessons from this study regard the fact that this is a cross-sectional research, and not a longitudinal one, thus not allowing for evaluation of dynamic impacts. A limitation is due to method employed to measure distance to parks. We have adopted the walking distance criteria, but also public transportation one should also be used in the accessibility measure. Moreover, our analysis is based on individual statements that cannot be checked, which would allow us a more objective assessment of the relationship between spatial proximity and psychological well-being, and we did not record any information about the access attitudes to green areas. Analogous remarks can be carried out with reference to subjects self-reporting their (perceived) diseases. It is therefore necessary to develop more sophisticated observation tools and devices to capture the needed information, whereas minimizing as far as possible informational noise. This is a much-needed improvement and an exciting challenge for the research to come.

Our analysis on green area is a post doc analysis since the original study [52], which has highlighted all the possible key factors which may be influential in the Milan case. The research was not intended to focus specifically on the issue of green areas in its relationship to well-being, but thanks to the evidence provided from survey and GIS correlation we have focused our attention on evaluating how spatial proximity of city dwellers to urban parks and gardens will affect subjective well-being. It was also beyond the scope of the paper to highlight all the possible key factors which may be influential in the Milan case, which are here reported only as feasible element of comparison between classical urban well-being determinant (as noted in Section 3) and spatial proximity. For it, we look forward to further studies. 
The literature review has highlighted an element that is not part of the aims of this study but plays a key role with respect to the analysis and evaluation of the possible effects of urban public spaces, such as parks and gardens, that is to say the lack of an effective evaluation model to understand the complexity of social interactions. We have the impression that if green areas can positively affect well-being, this occurs only when they are symbolically charged; mere proximity is not enough, as some classical studies suggest and our study confirms. This is a topic on which a lot of work is possible in the future. It thus emerges that, together with the need for a more accurate and scientific approach to the value of parks, gardens, and green areas for physical health and subjective well-being, an urban planning model is also required in order to define the possible rewards from urban green areas not only in terms of aesthetics but also in terms of quality of life for inhabitants. In this perspective, the engagement of people who take part in the definition of the urban context is necessary such as planners and administrators and also doctors, sociologists, and economists, for example, aiming to supply the best return in terms of quality of life of urban spaces and in particular the green urban environment, a fundamental element historically rooted in human development.

\section{Conflict of Interests}

The authors declare that there is no conflict of interests regarding the publication of this paper.

\section{References}

[1] G. MacKerron, "Mean streets, green streets: valuing urban environmental quality with spatial subjective wellbeing data," in Proceedings of the Sire Workshop on Well-being, Happiness and the Environment, University of Stirling, Stirling, Scotland, April 2010.

[2] R. Smyth, I. Nielsen, Q. Zhai et al., "A study of the impact of environmental surroundings on personal well-being in urban China using a multi-item well-being indicator," Population and Environment, vol. 32, no. 4, pp. 353-375, 2011.

[3] E. Grossi, A. Compare, C. Lonardi, R. Cerutti, E. Callus, and M. Niero, "Gender-related effect of cultural participation in psychological well-being: indications from the well-being project in the Municipality of Milan," Social Indicators Research, vol. 114, no. 2, pp. 255-271, 2013.

[4] A. Campbell, E. P. Converse, and W. L. Rodgers, The Quality of American Life: Perceptions, Evaluations, and Satisfactions, Russel Sage Foundation, 1976.

[5] A. Offer, The Challenge of Affluence: Self-Control and Well-Being in the United States and Britain Since 1950, Oxford University Press, Oxford, UK, 2006.

[6] P. L. Sacco and M. Viviani, "Scarsità, benessere, libertà nel contesto dell'economia dell'identità," Istituzioni e Sviluppo Economico, vol. 1, no. 3, pp. 5-41, 2003.

[7] R. Ryan and E. Deci, "On happiness and human potentials: a review of research on hedonic and eudaimonic well-being," in Annual Review of Psychology, S. Fiske, Ed., vol. 52, Palo Alto, Calif, USA, pp. 141-166, Annual Reviews Inc., 2001.

[8] M. Argyle, The Psychology of Happiness, Routledge, London, UK, 2001.
[9] C. L. Keyes, Flourishing, John Wiley \& Sons, New York, NY, USA, 2010.

[10] D. Kahneman and A. B. Krueger, "Developments in the measurement of subjective well-being," Journal of Economic Perspectives, vol. 20, no. 1, pp. 3-24, 2006.

[11] E. Lahtinen, Framework for Promoting Mental Health in Europe, STAKES, Helsinki, Finland, 1999.

[12] W. Pavot and E. Diener, "Happiness experienced: the science of subjective well-being," in Handbook of Happiness, Oxford University Press, Oxford, UK, 2013.

[13] B. S. Frey and A. Stutzer, Happiness and Economics: How the Economy and Institutions Affect Human Well-Being, Princeton University Press, 2010.

[14] E. Sandow, "Til work do us part: the social fallacy of longdistance commuting," Urban Studies, vol. 51, no. 3, pp. 526-543, 2014.

[15] E. Tovar and L. Bourdeau-Lepage, "Well-being disparities within the paris region: a capabilist spatialised outlook," Urban Studies, vol. 50, no. 8, pp. 1575-1591, 2013.

[16] K. Rehdanz and D. Maddison, "Climate and happiness," Ecological Economics, vol. 52, no. 1, pp. 111-125, 2005.

[17] E. Grossi, G. T. Blessi, P. L. Sacco, and M. Buscema, "The interaction between culture, health and psychological well-being: data mining from the Italian Culture and Well-Being Project," Journal of Happiness Studies, vol. 13, no. 1, pp. 129-148, 2012.

[18] S. T. Anderson and S. E. West, "Open space, residential property values, and spatial context," Regional Science and Urban Economics, vol. 36, no. 6, pp. 773-789, 2006.

[19] T. N. Clark, R. Lloyd, K. K. Wong, and P. Jain, "Amenities drive urban growth," Journal of Urban Affairs, vol. 24, no. 5, pp. 493$515,2002$.

[20] R. Florida, C. Mellander, and P. J. Rentflow, "The happiness of cities," Regional Studies, vol. 47, no. 4, pp. 613-627, 2011.

[21] E. Grossi, P. L. Sacco, G. T. Blessi, and R. Cerutti, “The impact of culture on the individual subjective well-being of the Italian population: an exploratory study," Applied Research in Quality of Life, vol. 6, no. 4, pp. 387-410, 2011.

[22] R. S. Ulrich, R. F. Simons, B. D. Losito, E. Fiorito, M. A. Miles, and M. Zelson, "Stress recovery during exposure to natural and urban environments," Journal of Environmental Psychology, vol. 11, no. 3, pp. 201-230, 1991.

[23] F. E. Kuo, "Coping with poverty impacts of environment and attention in the inner city," Environment and Behavior, vol. 33, no. 1, pp. 5-34, 2001.

[24] P. Apparicio, A.-M. Séguin, and D. Naud, “The quality of the urban environment around public housing buildings in Montréal: an objective approach based on GIS and multivariate statistical analysis," Social Indicators Research, vol. 86, no. 3, pp. 355-380, 2008.

[25] G. W. Evans, “The built environment and mental health," Journal of Urban Health, vol. 80, no. 4, pp. 536-555, 2003.

[26] C. Clark, B. Candy, and S. Stansfeld, "Mental health and the environment: a systematic review," Report, Mental Health Foundation, 2006.

[27] J. Punter, Ed., Urban Design and the British Urban Renaissance, Routledge, London, UK, 2009.

[28] P. L. Sacco and G. T. Blessi, "The social viability of culture-led urban transformation processes: evidence from the Bicocca District, Milan," Urban Studies, vol. 46, no. 5-6, pp. 1115-1135, 2009. 
[29] G. Tavano Blessi, E. Grossi, P. L. Sacco, G. Pieretti, and G. Ferilli, "Cultural participation, relational goods and individual subjective well-being: some empirical evidence," Review of Economics \& Finance, vol. 4, pp. 33-46, 2014.

[30] R. Kaplan and S. Kaplan, The Experience of Nature: A Psychological Perspective, CUP Archive, 1989.

[31] G. Burns, "The role of the natural environment in well-being," in The Science of Well-Being, F. Huppert, Ed., Oxford University Press (OUP), Oxford, UK, 2005.

[32] M. Pacione, "Urban environmental quality and human wellbeing-a social geographical perspective," Landscape and Urban Planning, vol. 65, no. 1-2, pp. 19-30, 2003.

[33] A. Chu, A. Thorne, and H. Guite, "The impact on mental wellbeing of the urban and physical environment: an assessment of the evidence," Journal of Mental Health Promotion, vol. 3, no. 2, pp. 8-17, 2004.

[34] Levett-Therivel Sustainability Consultant, Wellbeing: International Policy Interventions: Report to DEFRA, 2007, http:/gnhusa .org/wp-content/uploads/2012/11/DEFRA-Wellbeingresearchsynthesisreport.pdf.

[35] A. Gidlöf-Gunnarsson and E. Öhrström, "Noise and well-being in urban residential environments: the potential role of perceived availability to nearby green areas," Landscape and Urban Planning, vol. 83, no. 2-3, pp. 115-126, 2007.

[36] J. Pretty, J. Peacock, R. Hine, M. Sellens, N. South, and M. Griffin, "Green exercise in the UK countryside: effects on health and psychological well-being, and implications for policy and planning," Journal of Environmental Planning and Management, vol. 50, no. 2, pp. 211-231, 2007.

[37] B. Giles-Corti, M. H. Broomhall, M. Knuiman et al., "Increasing walking: how important is distance to, attractiveness, and size of public open space?" American Journal of Preventive Medicine, vol. 28, no. 2, pp. 169-176, 2005.

[38] R. Kaplan, "The nature of the view from home psychological benefits," Environment and Behavior, vol. 33, no. 4, pp. 507-542, 2001.

[39] Å. Lundgren-Nilsson, I. H. Ö. Jonsdottir, G. Ahlborg, and A. Tennant, "Construct validity of the psychological general well being index (PGWBI) in a sample of patients undergoing treatment for stress-related exhaustion: a rasch analysis," Health and Quality of Life Outcomes, vol. 11, no. 1, article 2, 2013.

[40] A. Shapiro and C. L. M. Keyes, "Marital status and social wellbeing: are the married always better off?" Social Indicators Research, vol. 88, no. 2, pp. 329-346, 2008.

[41] S. Penco, M. Buscema, M. Patrosso, A. Marocchi, and E. Grossi, "New application of intelligent agents in sporadic amyotrophic lateral sclerosis identifies unexpected specific genetic background," BMC Bioinformatics, vol. 9, article 254, 2008.

[42] M. Buscema, "Theory: foundations of artificial neural networks," Substance Use and Misuse, vol. 33, no. 1, pp. 17-199, 1998.

[43] C. Ambrey and C. Fleming, "Public green space and life satisfaction in urban Australia," Urban Studies, vol. 51, no. 6, pp. 1290-1321, 2014.

[44] G. T. Blessi, D.-G. Tremblay, M. Sandri, and T. Pilati, "New trajectories in urban regeneration processes: cultural capital as source of human and social capital accumulation. Evidence from the case of Tohu in Montreal," Cities, vol. 29, no. 6, pp. 397-407, 2012.

[45] UN DESA, World Urbanization Prospects: The 2009 Revision, Department of Economic and Social Affairs, New York, NY, USA, 2010.
[46] A. Antoci, P. L. Sacco, and P. Vanin, "Social capital accumulation and the evolution of social participation," Journal of SocioEconomics, vol. 36, no. 1, pp. 128-143, 2007.

[47] R. Easterlin, "Towards a better theory of happiness," in Economics and Happiness: Framing the Analysis, L. Bruni and P. L. Porta, Eds., pp. 29-64, Oxford University Press, Oxford, UK, 2005.

[48] L. Becchetti, G. Degli Antoni, and M. Faillo, "Let's meet up! The role of relational goods in promoting cooperation," Journal of Socio-Economics, vol. 39, no. 6, pp. 661-669, 2010.

[49] R. J. Sampson, Great American City: Chicago and the Enduring Neighborhood Effect, University of Chicago Press, Chicago, Ill, USA, 2012.

[50] M. L. Small, Villa Victoria. The Transformation of Social Capital in a Boston Barrio, University of Chicago Press, Chicago, Ill, USA, 2004.

[51] T. S. Nielsen and K. B. Hansen, "Do green areas affect health? Results from a Danish survey on the use of green areas and health indicators," Health \& Place, vol. 13, no. 4, pp. 839-850, 2007.

[52] E. Grossi, A. Compare, C. Lonardi, R. Cerutti, E. Callus, and M. Niero, "Gender-related effect of cultural participation in psychological well-being: indications from the well-being project in the municipality of Milan," Social Indicators Research, vol. 114, no. 2, pp. 255-271, 2013. 

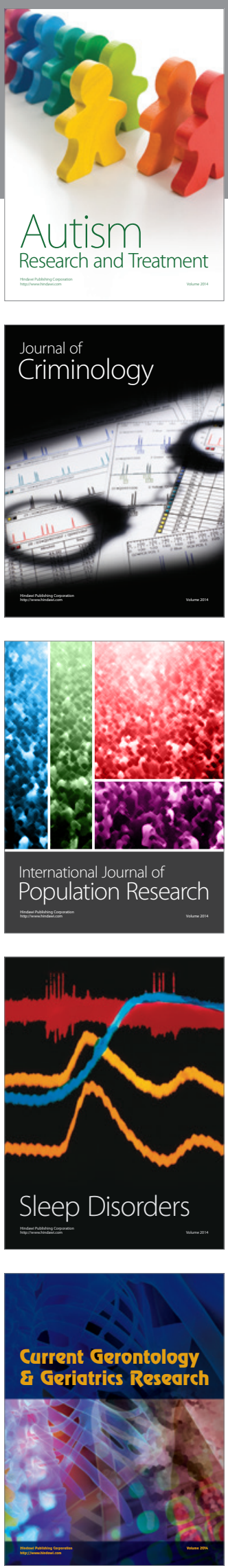
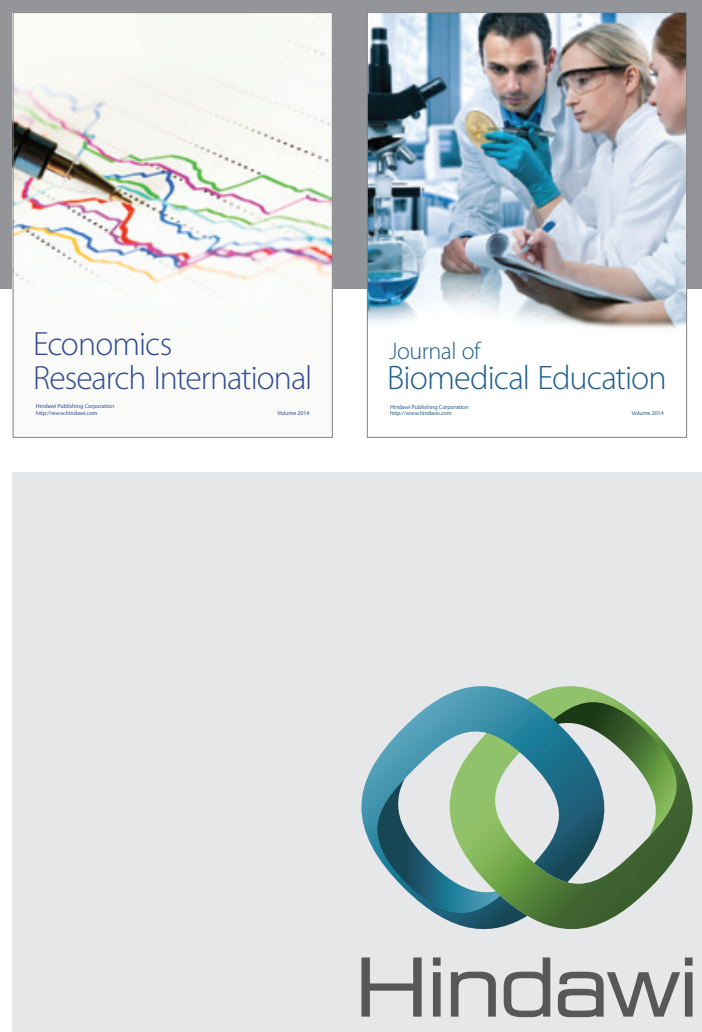

Submit your manuscripts at

http://www.hindawi.com
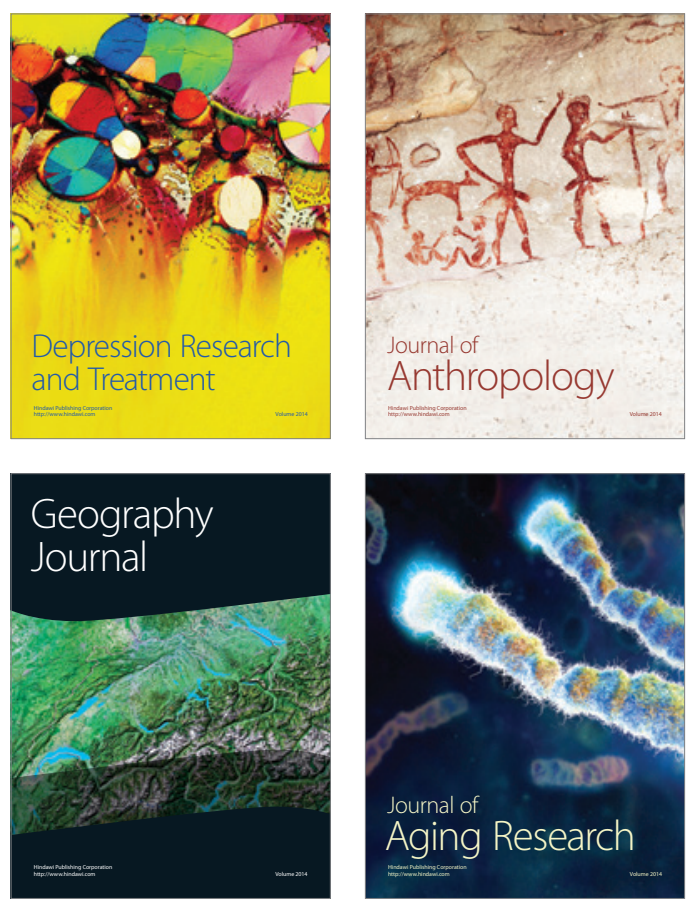
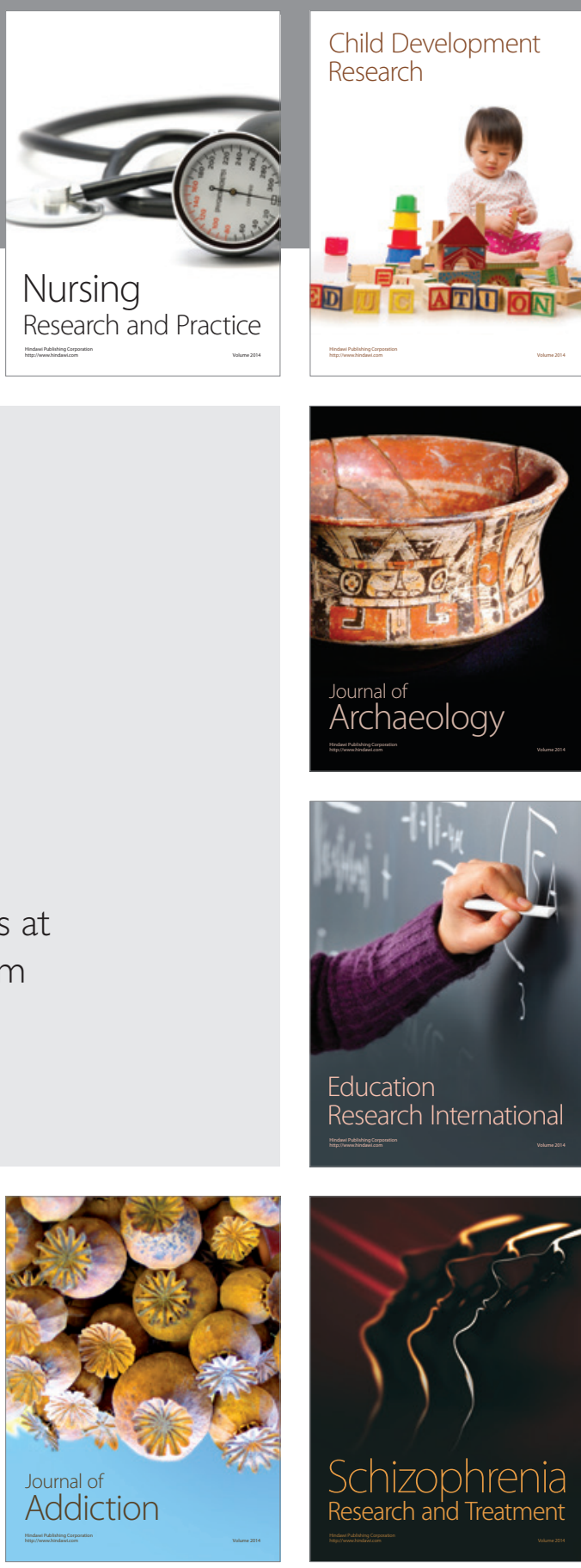

(D)
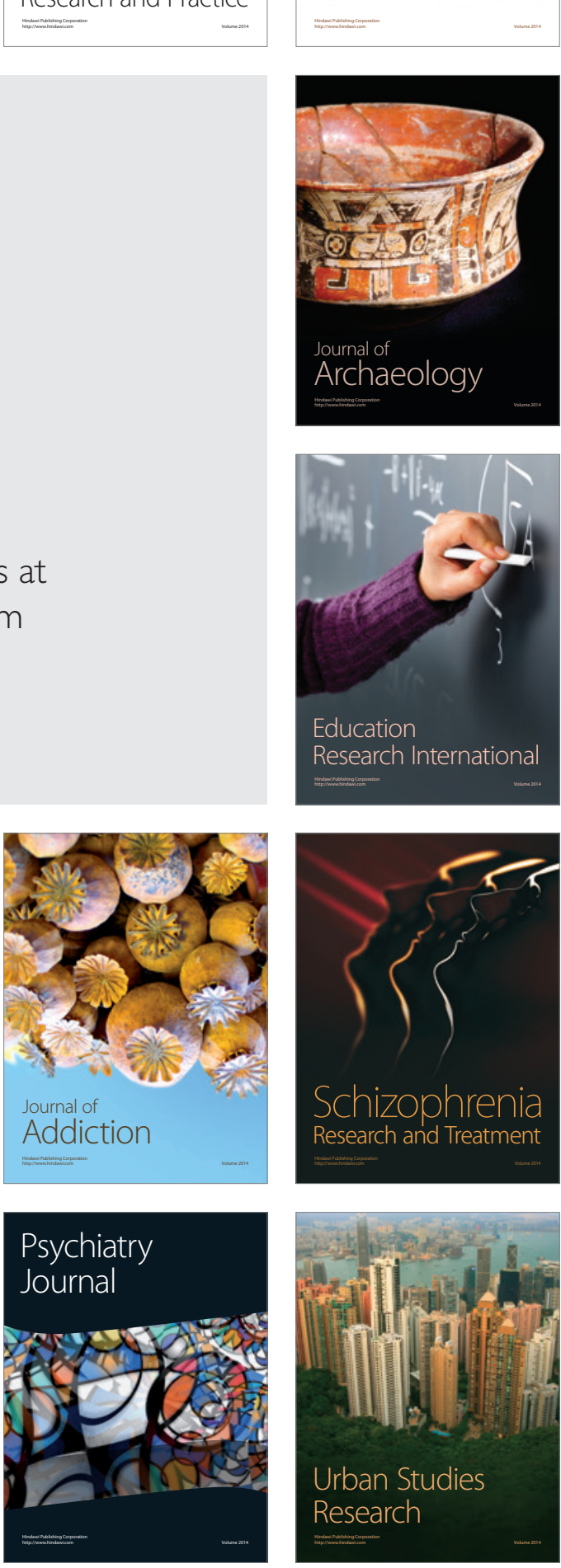